\title{
A Biological Restoration Model for Contaminated Coastal Marshes and Islands Using the Life Cycle of Acheta domesticus to Establish Environmental Sustainability
}

\author{
Anna Leyrer1, Edward Bush², James Geaghan33, Buffy Meyer ${ }^{4}$ \\ ${ }^{1}$ St. Joseph's Academy Baton Rouge, Louisiana, USA \\ ${ }^{2}$ Ag Center, Louisiana State University, Baton Rouge, LA, USA \\ ${ }^{3}$ Emeritus, Department of Experimental Statistics, Louisiana State University, Baton Rouge, LA, USA \\ ${ }^{4}$ Louisiana Department of Agriculture and Forestry, Baton Rouge, LA, USA \\ Email: acleyrer@gmail.com, ebush@agcenter.lsu.edu
}

How to cite this paper: Leyrer, A., Bush, E., Geaghan, J. and Meyer, B. (2020) A Biological Restoration Model for Contaminated Coastal Marshes and Islands Using the Life Cycle of Acheta domesticus to Establish Environmental Sustainability. Computational Water, Energy, and Environmental Engineering, 9, 13-21. https://doi.org/10.4236/cweee.2020.92002

Received: November 23, 2019

Accepted: January 17, 2020

Published: January 20, 2020

Copyright $\odot 2020$ by author(s) and Scientific Research Publishing Inc. This work is licensed under the Creative Commons Attribution International License (CC BY 4.0).

http://creativecommons.org/licenses/by/4.0/ (c) (i) Open Access

\begin{abstract}
Insects such as Acheta domesticus account for over half of marsh fish and migratory bird diet. Therefore, insects are very important to marsh and island ecosy stems and are susceptible to oil contamination, but little research has been done to study the effects of oil spills on cricket populations. The purpose of this experiment was to determine the effects of an oil spill event on Acheta domesticus mortality and hatch rate to establish environmental sustainability. The treatment groups included $0 \%$ oil contamination, $5 \%$ oil contamination and $10 \%$ oil contamination. The scientist counted the number of deaths in two-week, four-week, and six-week-old crickets after oil contamination. In addition, the scientist counted the number of hatchlings among treated cricket eggs. Error bar lines in the results section supported the scientist's hypothesis that the crickets would be affected by oil contamination. In fact, over $90 \%$ of the $10 \%$ oil treatment group died and over $80 \%$ of the $5 \%$ oil treatment group died after five days of oil contamination in the six-week-old experiment. The scientist used a Gas Chromatography Mass Spectrometer to determine if benzene, toluene, ethylbenzene, or xylene (total) were present in the sediment samples. BTEX exposure is suspected to possibly cause insect mortality. These volatile organic compounds were present in the samples and were found in higher quantities in samples with higher concentrations of oil. The highest quantity of volatile organic compounds present was xylene. After studying the population dynamics of the tested Acheta domesticus, the scientist found that the two-week and four-week-old crickets' survivability could
\end{abstract}


fit a linear model while the six-week-old crickets fit an exponential model. The scientist used these models to observe how long it took each treatment group to approach zero, and the oiled groups grew closer to a population of zero significantly faster. For example, it took about twenty-seven days for the $10 \%$ treatment group's population to approach zero whereas the control, $0 \%$ treatment group, took about seven days in the two-week-old cricket experiment. Overall, the six-week-old crickets had the highest mortality, and the cricket egg experiment resulted in extremely low hatch rate. Specifically, the highest number of crickets that hatched in an oiled replication either $5 \%$ or $10 \%$ oil was eight out of the five thousand eggs laid. Since the adult crickets are the most physically able to reproduce and the cricket eggs had low hatch rates, the cricket population would significantly decrease after an oil spill with $5 \%$ or more soil contamination. However, if the plant matter that crickets consume is still present in their environment, they may be able to recover.

\section{Keywords}

Entomology, Contamination, Oil, Insects, Sustainability, Marsh

\section{Introduction}

Arthropods play a crucial role in the environment contributing $20 \%-60 \%$ of the diet of estuarine fish and a large part of the migratory bird diet. In addition, insects like crickets (Ancheta domesticus) are useful bioindicators. Although an ecosystem can appear healthy, the lack of arthropods in an area can reveal that there is oil in the sediment. Often oil can remain in the sediment of marshlands for years following an oil spill event because marshes can be anoxic and low-energy. Therefore, it takes longer for oil-degrading bacteria to decompose the oil. An anoxic environment is one that is lacking oxygen, and a low-energy environment is one that has still water that does not bring in new nutrient rich sediment [1].

In the past decade, mineral revenues make up an average of $10 \%-15 \%$ of Louisiana's total revenue. The state is in an economic deficit partly because the oil prices have dropped. According to the US Energy Information Administration [2], "Louisiana's 18 oil refineries account for nearly one-fifth of the nation's crude oil-refining capacity." Although these statistics exemplify the importance of the oil industry to the state and the nation, the large petroleum, chemical, and natural gas industries in Louisiana cause the state to be one of the highest energy consumers in the US. Many living organisms die when oil spills occur, which is more often than most realize since many go unreported. In addition, there are natural oil seeps not caused by humans that can cause harm to insects and other wildlife.

Oil can kill crickets either from anoxia, from oil's lipophilic properties, or from volatiles released by oil. Lipophilic properties allow oil to dissolve lipids in the insect cuticle penetrating the nerves and the cytoplasm. Oil releases volatiles 
when it begins to degrade. These toxic gases are called BTEX, which stands for benzene, toluene, ethylbenzene, and xylene [3]. Crickets will most likely die from anoxia or oil's lipophilic properties when they are directly exposed or covered in oil, but crickets are most likely to die from BTEX when oil is in the sediment below them because the fumes released from BTEX are toxic for insects to assimilate. BTEX and other toxic chemicals such as chrysene and benzo(a)pyrene often resurface after storms cause coastal erosion re-exposing the ecosystem to contamination.

Although insects such as crickets have been found to be important to marsh ecosystems and affected by oil spills, they are relatively under researched in comparison to other organisms in these environments, so the scientist decided to study the effects of oil contamination on crickets at different stages of their life cycle. A cricket's life cycle is composed of three primary stages: egg, nymph, and adult. Crickets can hatch between 8 - 25 days and are considered adults about six weeks after hatching [4].

\section{Methods}

\subsection{Sampling Methods}

Four studies were initiated to determine the effect of the exposure of crude oil on crickets' survival at different stages of growth. The age groups included were eggs, younger nymphs (two-week-old), older nymphs (four-week-old), and adults (six-week-old). In the study on cricket eggs, hatch rate was recorded daily. In the two nymph studies and the adult study, mortality was recorded daily. Oil exposure treatments were as follows: $0 \%, 5 \%, 10 \% \mathrm{v} / \mathrm{v}$. There were five replications of each treatment group in each study.

Fifteen thousand cricket eggs donated by Ovipost and 3000 crickets (1000 adults, 1000 nymphs, and 1000 hatchlings) purchased by Fluker's Farms in Port Allen, Louisiana. Fluker's cricket quencher, Fluker's cricket food, peat moss, sand, and magnetic thermometer were utilized to raise crickets during the test. Optimum temperature ranges from $80^{\circ} \mathrm{F}-95^{\circ} \mathrm{F}$. BP crude oil from a Gulf of Mexico rig was used-acquired through Louisiana State University Department of Environmental Sciences.

To remove dead crickets daily, the scientists used protective gear such as gloves, a face mask, goggles, long pants, and sleeves to prevent crude oil exposure. The crickets were placed on a sheet of paper outside of their container to be counted on the plain background.

All research was completed in Baton Rouge, Louisiana during July and August 2018.

\subsection{Data Analyses}

The scientist recorded the percentage of daily hatch rate in the egg study and compared the treatment groups. In addition, the mortality of the adult and two nymphs groups were recorded daily. The scientist then performed an analysis of 
covariance on the adult and nymph data. The adult survivability fit a regression on an exponential model, but the two nymph studies fit best with a regression on a linear model. The estimation method used was restricted maximum likelihood (REML) (Table 1).

In addition, the scientist ran the sediment used as a substrate in the studies through a gas chromatography mass spectrometer to find if BTEX volatile organic compounds-benzene, toluene, ethylbenzene, and xylene-would be present. Sediment from all three treatment groups were tested.

\section{Results}

\subsection{Egg Acheta domesticus}

The control group that was placed in unoiled sediment with $0 \%$ contamination surpassed $20 \%$ hatch rate only four days after the first hatchling was spotted. In contrast, the hatch rate of cricket eggs planted in oiled sediment did not exceed $5 \%$ of the total amount planted (Figure 1).

\subsection{Two-Week-Old Acheta domesticus}

The two-week-old nymphs' survivability fit a linear model. The control- $0 \%$ oil contamination-group approaches zero at a much slower rate than the oiled groups. It would take about fourteen days for the $10 \%$ oil group to approach zero and about twenty days for the $5 \%$ oil group. Meanwhile, the control still had not approached zero after twenty days. The error bars suggest that in the first three days of the experiment the three treatment groups were statistically similar

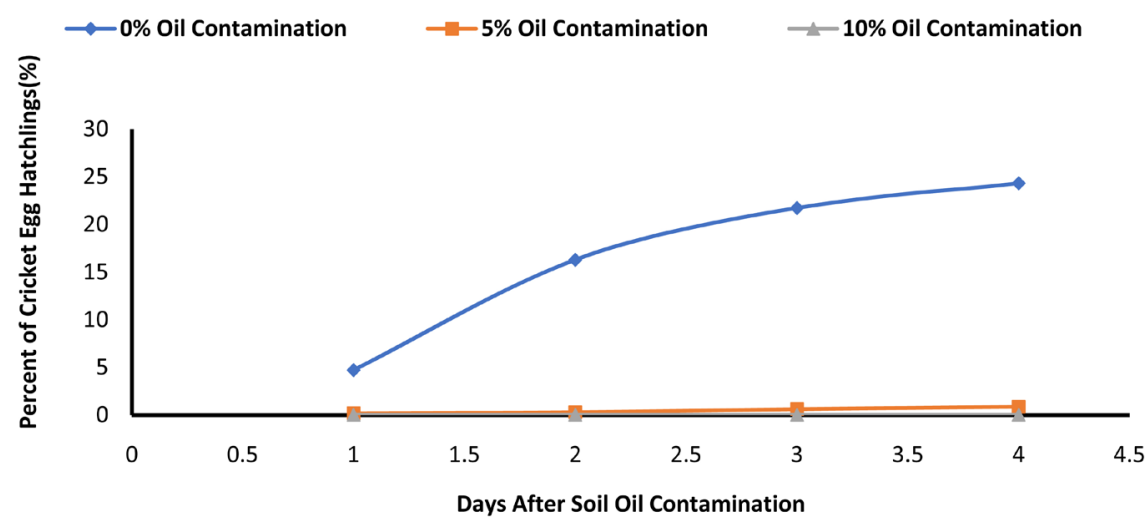

Figure 1. Percent of cricket eggs hatched after soil oil contamination treatments for a duration of four days.

Table 1. Formulas used to calculate cricket survivability after oil contamination.

\begin{tabular}{cccc}
\hline & Two Week Nymphs & Four Week Nymphs & Six Week Adults \\
\hline 0\% Oil Contamination & $\mathrm{y}=-0.9233 \mathrm{x}+50$ & $\mathrm{y}=-3.0822 \mathrm{x}+50$ & $\mathrm{y}=50 \mathrm{e}^{-0.271 \mathrm{x}}$ \\
$\mathbf{5 \% \text { Oil Contamination }}$ & $\mathrm{y}=-2.5667 \mathrm{x}+50$ & $\mathrm{y}=-3.7622 \mathrm{x}+50$ & $\mathrm{y}=50 \mathrm{e}^{-1.417 \mathrm{x}}$ \\
$\mathbf{1 0 \% \text { Oil Contamination }}$ & $\mathrm{y}=-3.83+50$ & $\mathrm{y}=-5.3756 \mathrm{x}+50$ & $\mathrm{y}=50 \mathrm{e}^{-1.817 \mathrm{x}}$ \\
\hline
\end{tabular}


to each other. Some error bars may be hidden by the data point (Figure 2). Since the Pr value was less than 0.05 , all treatments are statistically significant for this group (Table 2).

\subsection{Four-Week-Old Acheta domesticus}

The four-week-old nymphs' survivability also fit a linear model. The control- $0 \%$ oil contamination-group approaches zero at a much slower rate than the oiled groups. The $10 \%$ oil group would approach zero before a duration of ten days, the $5 \%$ oil group would approach zero before a duration of fifteen days, and the $0 \%$ oil group would approach zero before a duration of twenty days. The error bars suggest that the $0 \%$ and the $5 \%$ contaminated groups were statistically similar to each other for the first five days and similar to the $10 \%$ in the first three days. Some error bars may be hidden by the data point (Figure 3). Since the Pr value was less than 0.05 , all treatments are statistically significant for this group (Table 3).

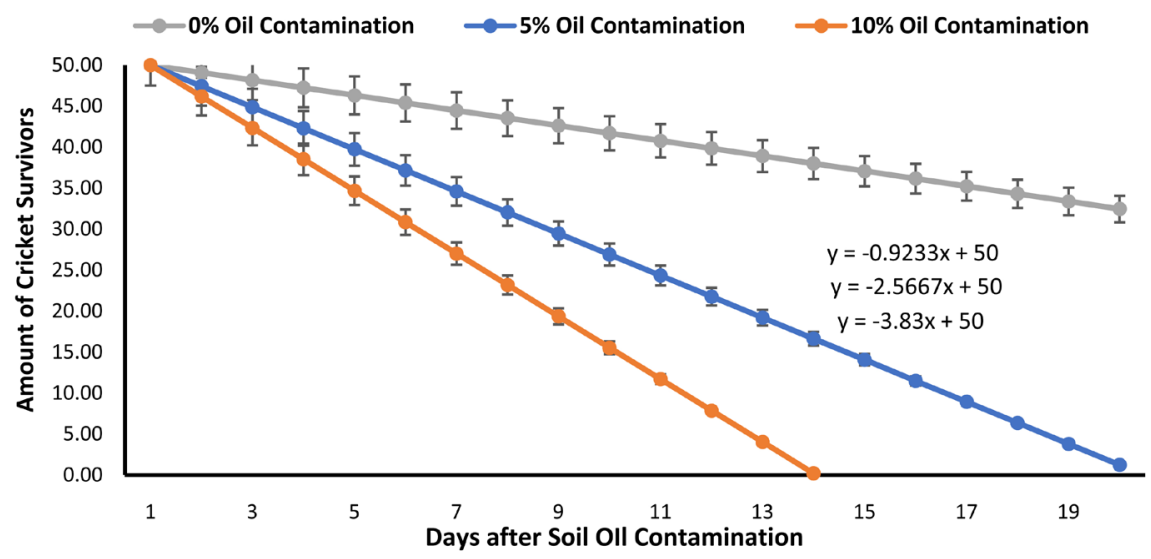

Figure 2. Survival of two-week-old crickets after soil oil contamination.

Table 2. Solution for fixed effects.

\begin{tabular}{ccccccc}
\hline Effect & Treatment & Estimate & Standard Error & DF & t Value & $\operatorname{Pr}>|\mathbf{t}|$ \\
\hline Intercept & & 3.9431 & 0.03563 & 71 & 110.67 & $<0.0001$ \\
Day * Treatment & $\mathbf{0 \%}$ & -0.05088 & 0.01878 & 71 & -2.71 & 0.0084 \\
Day * Treatment & $\mathbf{5 \%}$ & -0.1401 & 0.01878 & 71 & -7.46 & $<0.0001$ \\
Day * Treatment & & & & & & \\
& $10 \%$ & -0.2488 & 0.01878 & 71 & -13.25 & $<0.0001$ \\
\hline
\end{tabular}

Table 3. Solution for fixed effects.

\begin{tabular}{ccccccc}
\hline Effect & Treatment & Estimate & Standard Error & DF & t Value & $\operatorname{Pr}>|\mathbf{t}|$ \\
\hline Intercept & & 49.8267 & 0.9117 & 71 & 54.65 & $<0.0001$ \\
Day * Treatment & $\mathbf{0 \%}$ & -3.0822 & 0.4805 & 71 & -6.41 & $<0.0001$ \\
Day * Treatment & $\mathbf{5 \%}$ & -3.7622 & 0.4805 & 71 & -7.83 & $<0.0001$ \\
Day * Treatment & $\mathbf{1 0 \%}$ & -5.3756 & 0.4805 & 71 & -11.19 & $<0.0001$ \\
\hline
\end{tabular}




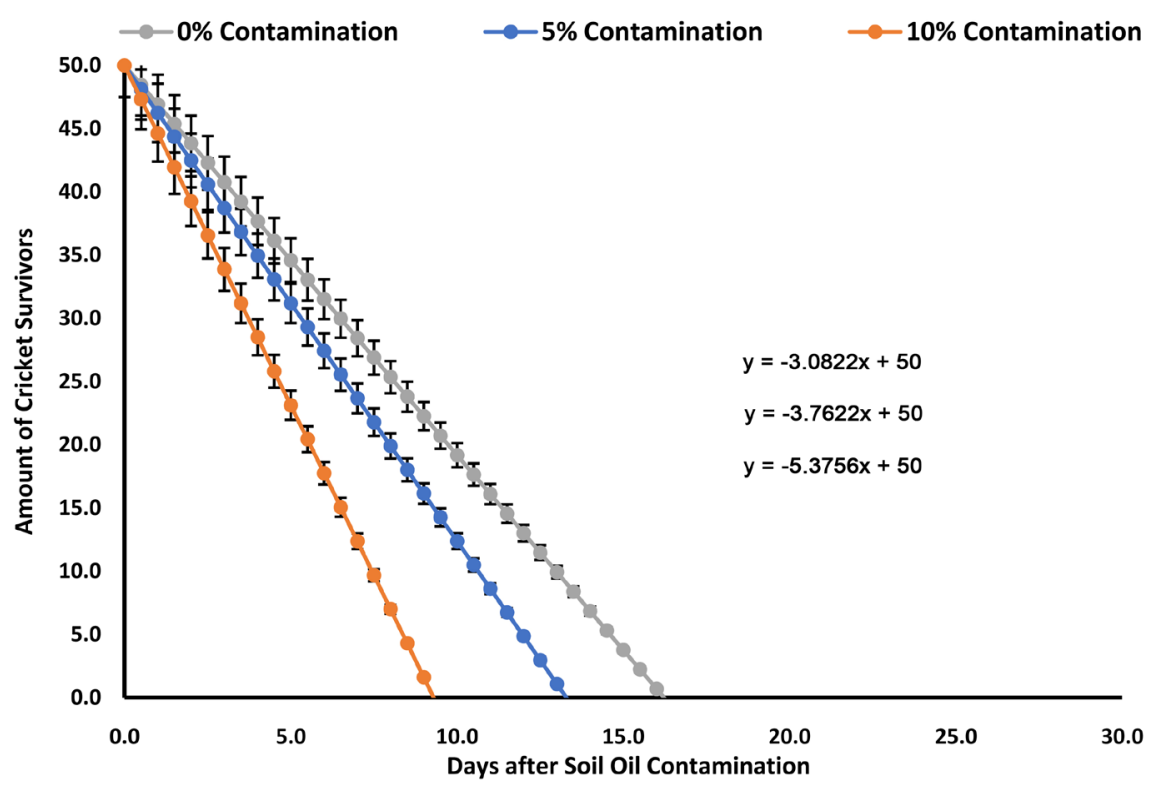

Figure 3. Survival of four week old crickets after soil oil contamination.

\subsection{Six-Week-Old Acheta domesticus}

The adult crickets' survivability fit an exponential model. Again, the control- $0 \%$ oil contamination-group approaches zero at a much slower rate than the oiled groups. Both the $5 \%$ and $10 \%$ treatment groups approached zero after only three days. Whereas the control group did not approach zero by day five. As shown by the error bars, the contaminated groups lacked any statistical similarity beyond the first day. Some error bars may be hidden by the data point (Figure 4). Since the Pr value was less than 0.05, all treatments are statistically significant except the control-0\% contamination-for this group (Table 4).

\subsection{BTEX Volatile Organic Compounds}

Since the oil proved to cause higher mortality in the nymph and adult studies and lower hatch rate in the cricket egg study, the scientist wanted to then find out what could possibly have caused cricket death. After testing the sediment substrate for BTEX volatile organic compounds, these compounds were found to be present and in higher quantities in higher concentrations of oil contamination. The compound found with the highest quantities was xylene. Some error bars may be hidden by the data point, but the quantities of benzene and ethylbenzene were similar at two data points at which they were touching. These were $4 \%$ and $7 \%$ (Figure 5).

\section{Discussion}

\subsection{Egg Acheta domesticus}

The oiled egg study showed that cricket egg germination or hatch rate is greatly affected by oil spill events. The hatchlings would be the best option for replenishing the population of crickets after loss of adult and nymph life. However, 


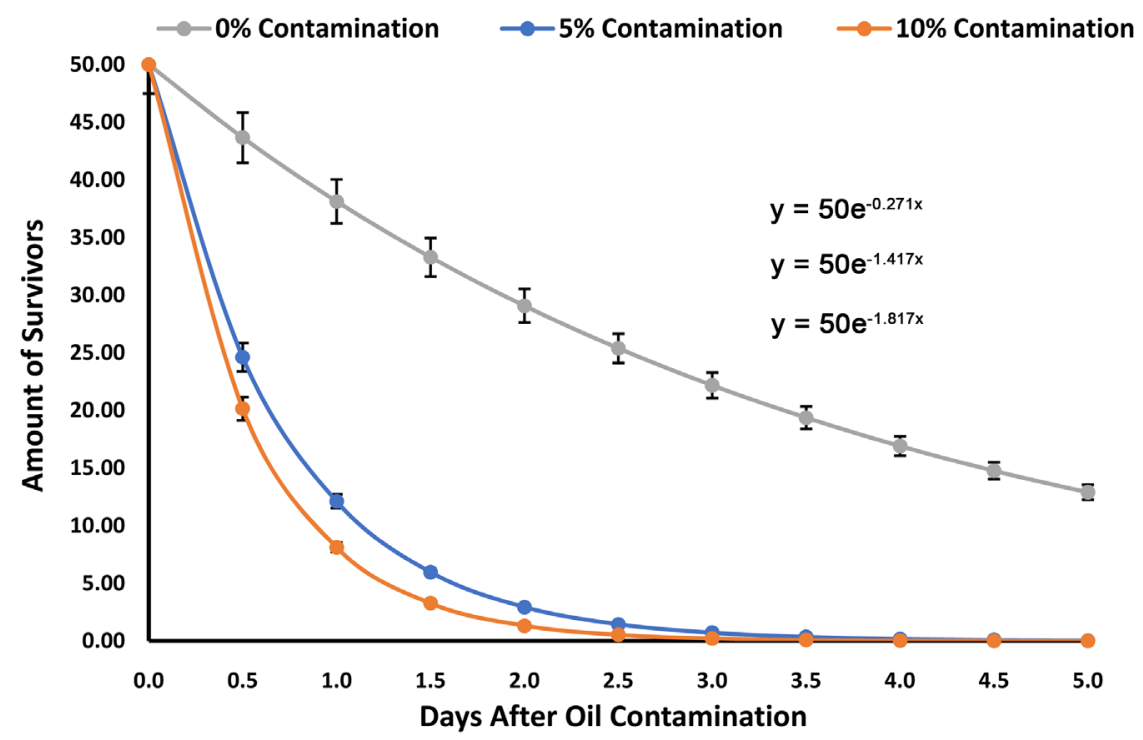

Figure 4. Adult cricket survival after oil contamination.

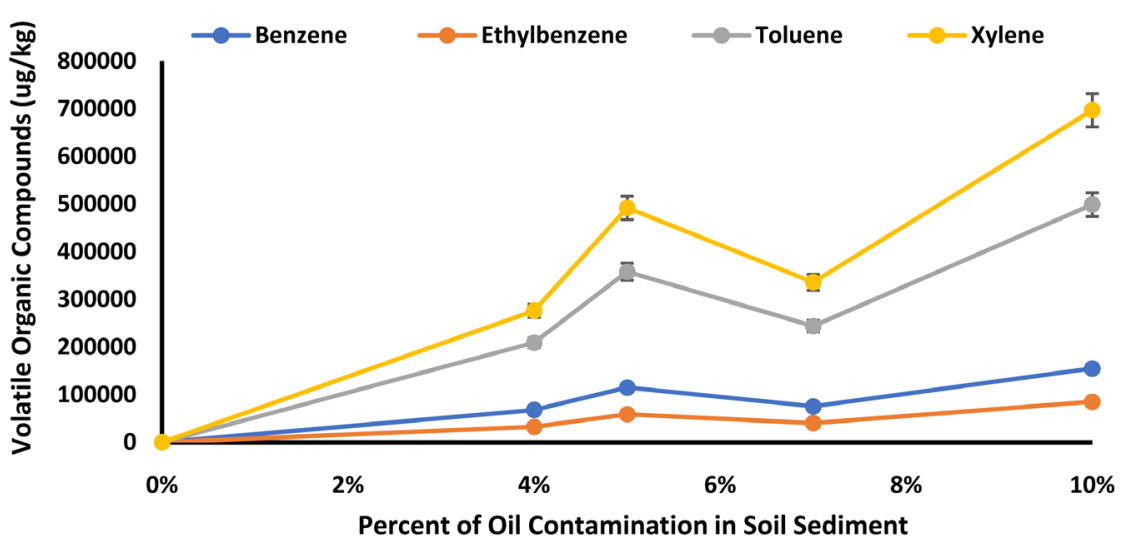

Figure 5. Volatile organic compounds detected in various oil concentrations.

Table 4. Solution for fixed effects.

\begin{tabular}{ccccccc}
\hline Effect & Treatment & Estimate & Standard Error & DF & t Value & $\operatorname{Pr}>|\mathbf{t}|$ \\
\hline Intercept & & 4.0175 & 0.577 & 116 & 6.96 & $<0.0001$ \\
Day * Treatment & $\mathbf{0 \%}$ & -0.271 & 0.1745 & 116 & -1.55 & 0.1231 \\
Day * Treatment & $\mathbf{5 \%}$ & -1.417 & 0.1745 & 116 & -8.12 & $<0.0001$ \\
Day * Treatment & $\mathbf{1 0 \%}$ & -1.8166 & 0.1745 & 116 & -10.41 & $<0.0001$ \\
\hline
\end{tabular}

the average hatch rate for the $10 \%$ oil contamination group was $0 \%$ of the planted cricket eggs; the $5 \%$ oil contamination group was statistically similar (Figure 1). If $5 \%$ or more of the volume of sediment of a marsh area or island is contaminated by oil, it is likely that scientists would have to reintroduce crickets into the environment after some attempt of remediation.

\subsection{Two-Week-Old Acheta domesticus}

Overall, the average survival of the two-week-old crickets was higher than the 
adult crickets' survival but lower than the four-week-old crickets' survival. This seems to suggest that younger nymphs are more susceptible to oil's harmful effects. Although many two-week-old crickets lack ovipositors which affects their ability to reproduce, the older nymphs and adults can reproduce [4]. If crickets cannot reach the older nymph and adult stages after oil contamination, the population will begin to approach zero since there are no eggs being produced.

\subsection{Four-Week-Old Acheta domesticus}

The four-week-old crickets had the lowest mortality and suspected highest survivability. The scientist theorizes that this because these older nymphs are young enough to fight the effects of oil contamination while old enough to have the needed anatomical developments to do so.

\subsection{Six-Week-0ld Acheta domesticus}

The oiled adult crickets lost over $50 \%$ of their population by day three. This is the highest average mortality recorded in the three studies that recorded mortality. As crickets at this stage are the most able to reproduce, the high mortality suggests little expected sustainability of the population. In fact, when looking at the adult and two-week-old mortality in conjunction with low average oiled hatch rate, the cricket population would be most likely to recover after oil contamination if crickets were reintroduced, and the plant matter they eat is unaffected by the oil contamination.

\subsection{BTEX Volatile Organic Compounds}

BTEX is suspected to be a cause of death for crickets, and this is supported by previous research. The presence of BTEX in the sediment substrate samples provides a basis for future research on oil-related insect death; specifically, which BTEX compound is more dangerous to insects compared to the others.

\section{Conclusions}

Previous research on this topic did not address multiple stages of the life cycle of a specific species. Often, scientists focused on varying the species of contaminated insects studied. Although this provides useful information, observing the effects of contamination on an entire life cycle can help scientists discuss the viability and sustainability of an affected ecosystem.

This study provides evidence for the harmful effects of oil contamination on one species at different stages of its lifecycle and underlines the importance of studying insects after oil spill events. Through this, one can observe that contamination can affect organisms of the same species in the same environment differently depending on the stage of the life cycle of the organism. This research provides a biological model for the impact of oil spill events on an insect population and, in turn the food web, as insects are the main food source for many animals. In addition, BTEX monitoring of contaminated sediment can provide 
insight into the potential viability of impacted wetlands.

\section{Acknowledgements}

Dr. Edward Bush, Dr. Buffy Meyer, Dr. Linda Hooper-Bui, Dr. James Geaghan, Ovipost, Fluker's Farms, Linda Messina, Jaqueline Savoia, GCAL LLC.

This manuscript is dedicated to the late Dr. James Geaghan.

\section{Conflicts of Interest}

The authors declare no conflicts of interest regarding the publication of this paper.

\section{References}

[1] Pennings, S., McCall, B. and Hooper-Bui, L. (2014). Effects of Oil Spills on Terrestrial Arthropods in Coastal Wetlands. BioScience, 64, 789-795.

http://www.jstor.org/stable/90006939

https://doi.org/10.1093/biosci/biu118

[2] US Energy Information Administration-EIA-Independent Statistics and Analysis. (n.d.). https://www.eia.gov/state/analysis.php?sid=LA

[3] Schleifstein, M. (2012) Gulf of Mexico oil Spill Reseachers Look into Effects on Wildlife, Ways to Improve Response. The Times-Picayune.

http://www.nola.com/news/gulf-oil-spill/index.ssf/2012/04/scientists_find_cause_fo r_conc.html

[4] Miller, H. (2004) Cricket. Kid Haven Press, Michigan. 